\title{
Reconstruction of Lost Fragments of Signal Received from the Wireless Sensor Measuring Fluid Pressure inside the Eye Ball
}

\author{
K. MuraWski ${ }^{a, *}$, L. GRAD ${ }^{a}$ AND M. RęKAS ${ }^{b}$ \\ ${ }^{a}$ Military University of Technology, Institute of Teleinformatics and Automatics, \\ S. Kaliskiego 2, 00-908 Warsaw, Poland \\ ${ }^{b}$ Military Institute of Medicine, Ophthalmology Department, Szaserów 128, 04-141 Warsaw, Poland \\ (Received May 12, 2014; in final form April 24, 2015)
}

The aim of the paper is to present a technique of reconstructing data received from a wireless sensor for measuring intraocular pressure. The algorithm was developed to automatically regenerate samples lost due to fortuitous events in clinical trials. The test consisted in measuring the pressure within $24 \mathrm{~h}$, during which the intraocular pressure curve were determined. The paper also discusses the structure and the physical basis of sensor operation and shows the advantages resulting from its use.

DOI: $10.12693 /$ APhysPolA.127.1596

PACS: 07.07.Df, 88.80.ht, 87.85.Ng

\section{Introduction}

Tonometry (the measurement of intraocular pressure) is a test performed during routine ophthalmic examination. In the practice of ophthalmology it is one of the useful diagnostic methods along with imaging methods [14]. Tonometry is of great importance in the diagnosis of glaucoma [5,6], ocular hypertension [7] or in the diagnosis of incidental increases in intraocular pressure after eye surgery [8]. Clinical symptoms are also indication for measuring intraocular pressure. Persistent conjunctival hyperemia or chronic ocular surface inflammation $[9,10]$ should especially be paid attention to. The intraocular pressure measurement is based on the principles of fluid mechanics [11]. The construction of the eyeball can be successfully modelled as a sphere filled with liquid [12]. The fluid pressure in the eyeball is kept at a constant level, in physiological conditions of $10 \mathrm{~mm} \mathrm{Hg}$ to $21 \mathrm{~mm} \mathrm{Hg}$, thanks to the prevailing equilibrium between the production of aqueous liquid and its outflow out of the eyeball. Outflow is carried out by conventional means through Schlemm's canal and by unconventional means through the fluid-filled spaces of the ciliary body, choroid and sclera. The method of measuring the intraocular pressure is based on the Imbert-Fick law. According to this law, by knowing the force applied perpendicularly to the surface of the sphere and the area undergoing deformation (flattening) the $p$ pressure inside the ball (the eyeball) can be determined. This pressure is transferred to the rigid walls of the eyeball, and also to any fluid cross-sections, perpendicular to these walls and the cross-sections at every point. Assuming that any segment of the surface of the eye may be represented by

* corresponding author; e-mail: k.murawski@ita.wat.edu.pl the $\boldsymbol{S}$ vector, whose value is equal to the segment surface area, and the direction is perpendicular as well as facing outward from the surface, the value of the $\boldsymbol{F}$ force exerted on the $\boldsymbol{F}=p \boldsymbol{S}$ section can be determined. Since the direction of the $\boldsymbol{F}$ force and $\boldsymbol{S}$ vector are identical, Fig. 1a, the intraocular pressure value is determined from the formula $p=\boldsymbol{F} / \boldsymbol{S}$.

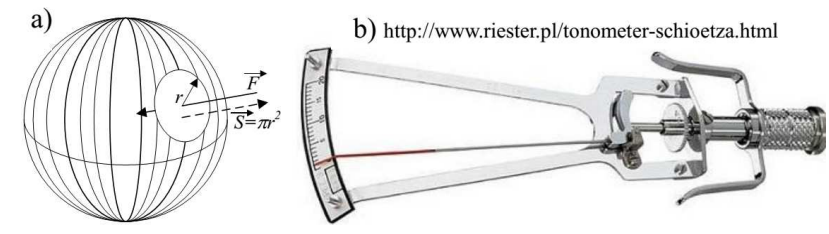

Fig. 1. Intraocular pressure measurement device: interpretation (a), tonometer (b).

In a broader sense the $p$ pressure of the fluid can vary depending on the choice of measurement of the surface of the sphere. This is due to the fact that the density $\rho$ of a homogeneous fluid $\rho$ (mass divided by volume) may depend on temperature and pressure. In case of the eye, an intraocular pressure measurement error resulting from this dependence is ignored since the main component of the aqueous liquid is water, whose density, even at significant changes in pressure and temperature, changes slightly $\left(1.000 \times 10^{3} \mathrm{~kg} / \mathrm{m}^{3}-\right.$ at $0{ }^{\circ} \mathrm{C}$ and $1 \mathrm{~atm} ; 0.958 \times 10^{3} \mathrm{~kg} / \mathrm{m}^{3}$ at $100^{\circ} \mathrm{C}$ and $1.0 \mathrm{~atm}$ as well as $1.002 \times 10^{3} \mathrm{~kg} / \mathrm{m}^{3}$ at $0^{\circ} \mathrm{C}$ and $\left.50 \mathrm{~atm}\right)$.

\section{Techniques to measure fluid pressure inside the eyeball}

Intraocular pressure measurement can be performed in many ways. One of the basic methods is by using the Schiöetz tonometer (impression tonometry) [13]. The test consists in exerting pressure on the cornea using a weight with a mass of $55 \mathrm{~g} .75 \mathrm{~g}, 100 \mathrm{~g}$, and 
$150 \mathrm{~g}$ weights are also used in this method. A pressed cornea deforms to an extent dependent on intraocular pressure. The size of the deformation is read from a scale. The test result is the basis for calculating the intraocular pressure. Currently, the most widely used technique is the Goldmann applanation tonometry [14]. During the test, the doctor presses the tip of the tonometer onto the cornea until it obtains two semicircles from its surface in the shape of an S. The intraocular pressure value can be determined by knowing the area and pressure force. Another way of measurement uses the pressure exerted on the cornea by a blast of air (noncontact tonometry) [15]. The air stream flattens the surface of the cornea. The pressure is determined analogously as in applanation tonometry - in accordance with the Imbert-Fick law.

There are also other measurement techniques available. Triggerfish lenses were used in the experiment conducted at the Military Institute of Medicine, Fig. 2a. Intraocular pressure of the eye is determined by measuring deformation of the contact lens adjacent to the wall of the eyeball, Fig. 2b.

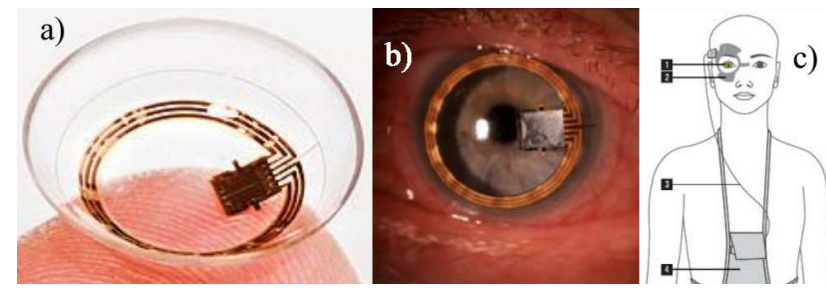

Fig. 2. Triggerfish measurement lens (a), during use (b), system elements (c) [16].

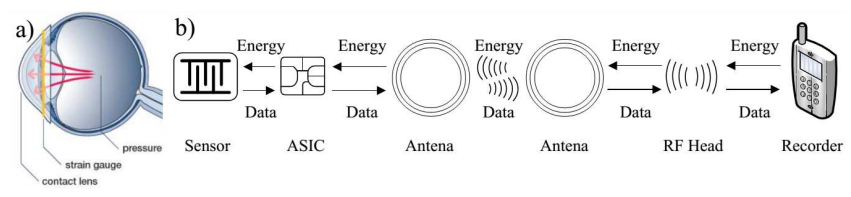

Fig. 3. Principle of operation of the measurement lens (a), measurement and measured signal transmission system (b) [17].

The deformation size is measured by a tensometer applied to the surface of the lens, Fig. 3a. The tensometer operates in a balanced Wheatstone bridge setup with the following parameters: the sensor resistance $R=3.2 \mathrm{k} \Omega \pm 4 \%$, voltage supply $U_{I}=1.5 \mathrm{~V}$. The deformation of the measuring lens disrupts the balance of the measuring bridge. The size of deformation is measured as a voltage signal with an amplitude of $\pm 35 \mathrm{mV}$. The voltage is then amplified and sampled at a frequency of $f=25 \mathrm{~Hz}$ through a 14 bit ADC converter. The digital value is sent to the recorder via radio at a frequency of $f=27 \mathrm{MHz}$ with a FSK modulation, Fig. 3b. The signal is received by the antenna placed near the eye. It is stored in digital form. An example of a test result before and after the Canaloplasty operation is presented in Fig. 4.
The course recorded in the logger consists of 288 samples stored every $5 \mathrm{~min}$. The adopted organization of recording covers a $24 \mathrm{~h}$ recording period. Each sample is determined from the median value of 300 measurements done in $30 \mathrm{~s}$ intervals, that corresponds to sampling at a frequency of $f=10 \mathrm{~Hz}$ [18]. The example shown in Fig. 4 presents the complete course of measurement. Less favourable situations were also observed in the studies, in which part of the measuring points have been lost. By analyzing the available records, it was ascertained that the lens worked properly. The main cause of data loss was communication errors. The numbers present as follows. Every 87000 measurements done during a single study about a thousand parity errors occurred (1\% CheckSumError), in 474 cases $(0.5 \%)$ it was not possible to establish communication (RxTimeout), and in 723 data transmission sessions $(0.83 \%)$ the signal value was considered low (SignalRectLow). The total percentage of transmission affected by error or so-called uncertain conditions (SignalRectLow) does not exceed 3\%. However, the overlapping of unfavourable conditions caused the occurrences of time gaps in the recorded signal.

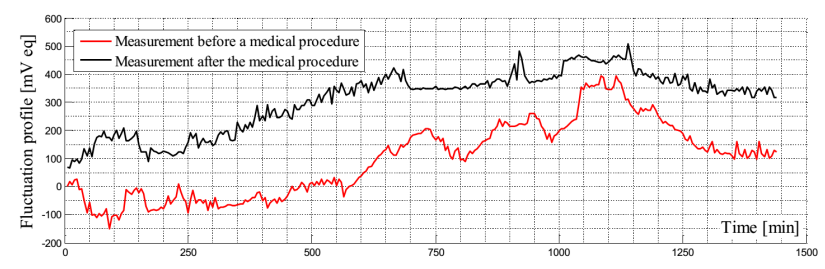

Fig. 4. Results of measuring eyeball deformation during a period of $24 \mathrm{~h}$.

The longest observed pause during recording was 150 min, Fig. 5. In practice, the fading of signals prevents inference on the basis of wave forms recorded before and after the operation including Canaloplasty. This fact has prompted the authors to develop a signal reconstruction algorithm presented in point 3 .

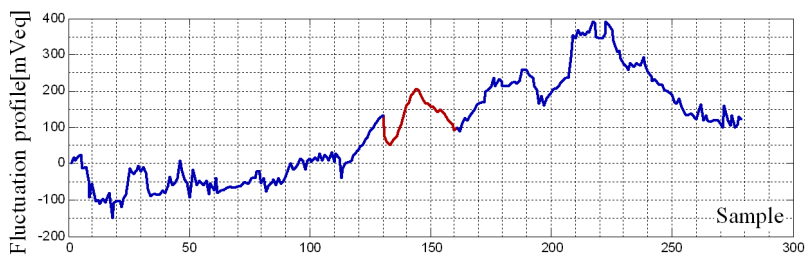

Fig. 5. Result of reconstruction for an interval width of $L=30$ and a prediction order of $p=18$.

\section{Signal reconstruction technique}

The reconstruction algorithm fills the gaps in the signal resulting from errors occurring during the radio transmission of data. This problem is solved through methods of interpolation. The paper presents an algorithm supplementing data that maintains the nature of the original signal. Thanks to this results are more reliable, Fig. 6, compared to the results of linear interpolation. 


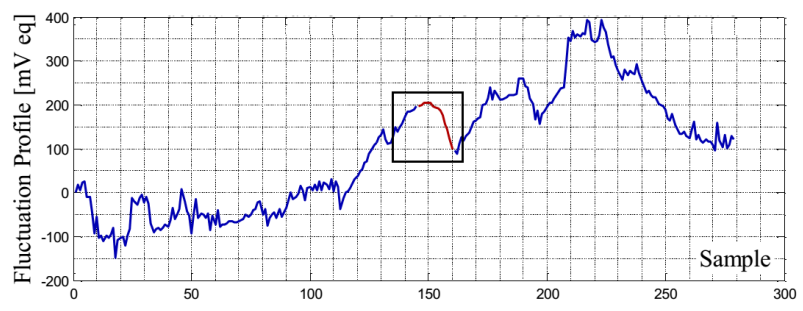

Fig. 6. Eyeball deformation measurement during the period of $24 \mathrm{~h}$ with an indicated short break and reconstruction result. Break duration: 90 min (18 samples).

The proposed reconstruction method assumes the existence of a correlation in the recorded signal. It is based on the AR autoregression model [19, 20]. The interpolation is realized in it by the combination of two extrapolations: "forward" and "backward". Forward extrapolation determines prediction models based on the left-side of the reconstructed interval. These models are used to obtain a prediction based on the left-side of the reconstructed section. Similarly, "backward" extrapolation is carried out on the right side of the reconstructed interval. The number of predictive models for each extrapolation corresponds to the length of the reconstructed interval. A single model is used to estimate the value of one of the missing samples. The idea of the presented method is illustrated in Fig. 7. This differs from the typical use of the AR model, where extrapolation is performed by a model implementing iterative reconstruction of missing samples (sample after sample).

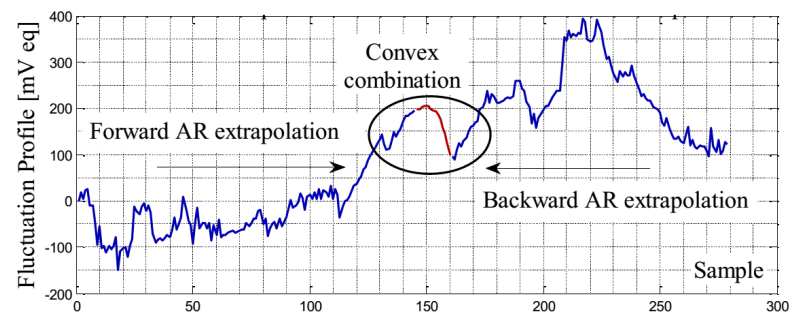

Fig. 7. The idea of the proposed method of interpolation.

A limitation of such an approach is moving the prediction error from the output to the input. As a result, a rapid growth of prediction error is attained. This is particularly disadvantageous when reconstructing a large number of samples. The executed experiments have shown that it can even prevent correct reconstruction of the signal $[20,21]$. The adopted AR model is defined according to (1):

$$
x(k)=\sum_{i=1}^{p} w(i) x(k-i)+e(k) .
$$

In Eq. (1) the individual elements are indicated as follows: $p$ - order of prediction, $w(i)$ - model factors, $e(k)$ - prediction error, $x(k)$ - signal sample. The value of $x(k)-e(k)$ is an estimation of the signal sample value at the moment of $k$. The $w(i)$ factors of the model are determined minimising prediction errors $e(k)$.
The proposed method simultaneously assumes the usage of many models (1) achieving a set of models with a cardinality of $L$ in the form of (2):

$$
\begin{gathered}
x(k)=\sum_{i=1}^{p} w_{1}(i) x(k-i)+e_{1}(k), \\
x(k+1)=\sum_{i=1}^{p} w_{2}(i) x(k-i)+e_{2}(k), \\
\ldots \\
x(k+L-1)=\sum_{i=1}^{p} w_{L}(i) x(k-i)+e_{L}(k) .
\end{gathered}
$$

Every model determines the partial prognosis of the signal value for the sample in the reconstructed interval. Interpolation of all samples in the proposed method is implemented by submitting "forward" and "backward" extrapolation in the form of a convex combination (3):

$$
y(l)=a(l) y_{f}(l)+(1-a(l)) y_{r}(l), \quad l=1,2, \ldots L
$$

In Eq. (3) the following indications were adopted: $L$ width of the reconstructed range, $y_{f}(l)$ - the result of forward extrapolation, $y_{r}(l)$ - result of backward extrapolation, $a(l)$ - combination factors, $0 \leq a(l) \leq 1$. $w(i)$ factors of the AR model can be determined using various methods aiming to minimise the error $e(k)$. The method of least squares and the iterative gradient Widrow-Hoff method [21] were used in the study to train linear neural networks. The Widrow-Hoff method was used when the method of least squares could not be used due to the peculiarities of the matrix.

\section{Results of research}

A series of simulation experiments were carried out in the study. They involved the reconstruction of the signal in ranges, for which the actual signal values were known. This made it possible to evaluate the proposed method. The results were referred to results of the reconstruction using linear interpolation (zero-order polynomial). Figures $5,8,9$ show the results at different stages of the algorithm. Forward AR extrapolation results are shown

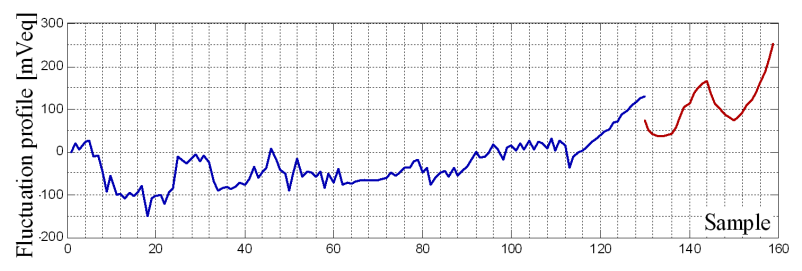

Fig. 8. "Forward" extrapolation for an interval width of $L=30$ and a prediction order of $p=20$.

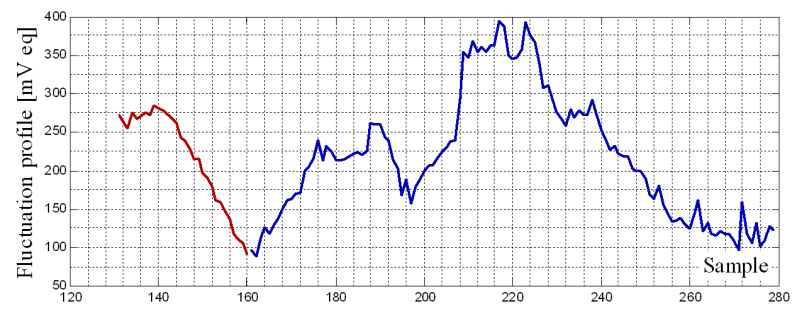

Fig. 9. "Backward" extrapolation for an interval width of $L=30$ and a prediction order of $p=18$. 
in Fig. 8. Figure 9 shows the progress achieved during backward AR extrapolation. The reconstructed signal samples are marked in red. It is worth noting that the two projections clearly maintain the nature of signals used for preparing models. The result of submitting both prognoses, the reconstruction of the missing part of the signal, is illustrated in Fig. 5.

While verifying the operation of the proposed method of signal reconstruction, the obtained result was compared to the original course and the result of linear interpolation, Fig. 10. The original signal is marked in blue (dotted line). The green colour (solid line) presents the result of linear interpolation. The result of the operation of the proposed method is indicated in red (dashed line).

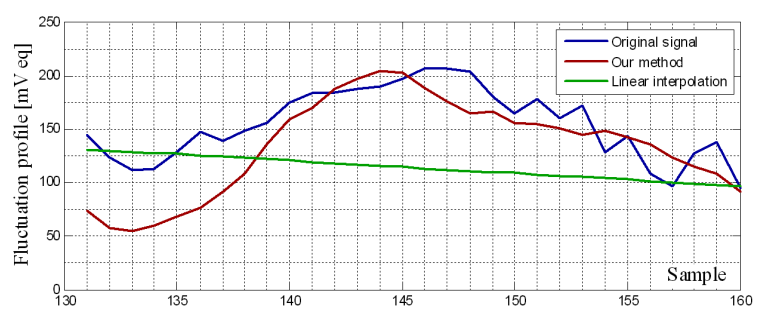

Fig. 10. Reconstructed signal and the result of linear interpolation against the original signal.

A satisfactory result of reconstruction was achieved in the presented example. The proposed technique maintained the nature of a signal simultaneously achieving an acceptable level of prognostic error. The prognostic error were calculated as the average relative deviation from the following equation: $(1 / L)\left[\sum_{i=1}^{L}(|y(i)-x(i)| / x(i))\right] 100 \%$. For the proposed technique level of prognostic error was equal to $19.9 \%$, for a linear interpolation of $25.4 \%$. The test was repeated for other real signals, in which loss of samples was simulated. The average relative deviation was assumed as an indicator of reconstruction error. It was calculated based on actual data. Results were compared with linear interpolation. The averaged results of 20 experiments are presented in Fig. 11.

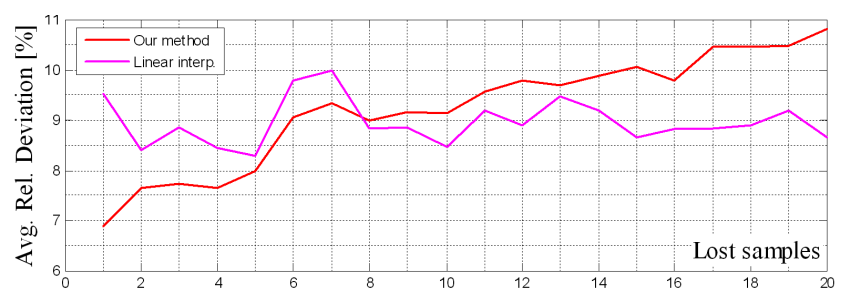

Fig. 11. The obtained reconstruction error as a function of the size of the reconstructed interval.

In the case of linear interpolation, reconstruction error, measured with an average relative deviation, was approximately constant and fluctuated around $9 \%$. In the proposed method of reconstruction the error increased along with the number of samples being reconstructed. It fits in the range of about $7 \%$ when reconstructing one sample to approximately $11 \%$ at 20 samples.

The proposed method at reconstructing 8 samples (40 min break from recording) is characterised with a lower prediction error than the linear interpolation while simultaneously maintaining the nature of the signal. At higher numbers of samples the prognostic error is slightly higher than the linear interpolation error (by about $3 \%$ for 20 samples).

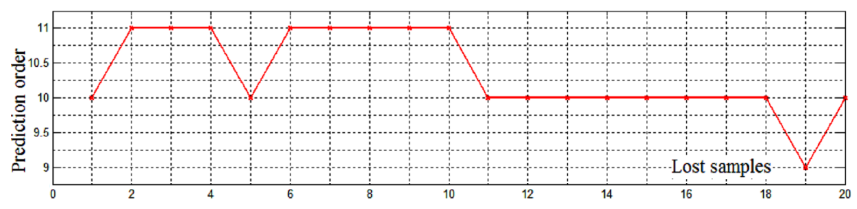

Fig. 12. Prediction order of models used for reconstruction from Fig. 11 in the function of the number of reconstructed signal samples.

During the experiments the impact of value of the prediction order on the signal reconstruction result were tested as well. For each reconstructed interval there were made series of experiments. The prediction order values for which the best results of reconstruction were achieved, are presented in Fig. 12. The prediction rate fluctuated insignificantly (from 9 to 11) during the reconstruction of the intervals with lengths from 1 sample to 20 samples, which considerably facilitates the use of the method.

\section{Conclusions}

The proposed method of reconstruction allows achieving correct results in the case of having a sufficient number of samples to learn the AR model. Application of the discussed method is difficult, if the lost samples are located near the beginning or end of the signal. In extreme cases this can lead to reconstruction only based on the forward or backward extrapolation. Learning the model can then be performed using techniques of non-linear interpolation or artificial intelligence [22].

\section{Acknowledgments}

This work has been supported by Military Institute of Medicine (M.R.) and by the Military University of Technology (K.M. and L.G.).

The work was presented at the 8th Conference Integrated Optics - Sensors, Sensing Structures and Methods, IOS'2014 sponsored by Polish Academy of Sciences. The conference was organized by the Committee of Electronics and Telecommunication at the Polish Academy of Sciences in cooperation with the Upper Silesian Division of the Polish Acoustical Society and Photonic Society of Poland, as well as the Department of Optoelectronics at the Silesian University of Technology. 


\section{References}

[1] K. Murawski, R. Różycki, P. Murawski, A. Matyja, M. Rękas, Acta Phys. Pol. A 124, 517 (2013).

[2] K. Różanowski, K. Murawski, Acta Phys. Pol. A 124, 558 (2013).

[3] K. Różanowski, K. Murawski, Acta Phys. Pol. A 122, 874 (2013).

[4] K. Murawski, K. Różanowski, Acta Phys. Pol. A 124, 509 (2013).

[5] Guidelines for Glaucoma, The Japan Glaucoma Society, Vol. 2, Tokyo 2006.

[6] http://www.nice.org.uk/nicemedia/live/12145/ 43792/43792.pdf, 2014.

[7] J.H. Shaver, J.H. Shen, A.L. Walker, 26th Ann. Int. Conf. IEEE Eng. Med. Biol. Soc., Vol. 1, San Francisco 2004, p. 2330.

[8] M. Fingeret, Primary Care Optometry News 85, 139 (2004).

[9] A. McDermott, V. Perez, A. Huang, S. Pflugfelder, M. Stern, C. Baudouin, R. Beuerman, A. Burns, V. Calder, M. Calonge, J. Chodosh, D. Coster, R. Dana, L. Hazlett, D. Jones, S. Kim, E. Knop, D. Li, B. Mitchell, J. Niederkorn, E. Pearlman, K. Wilhelmus, E. Kurie, The Ocular Surface 3, 11 (2005).

[10] H. Brewitt, Research Projects in Dry Eye Syndrome, Vol. 45, Karger, Hannover 2010.

[11] http://en.wikipedia.org/wiki/Intraocular_pressure.
[12] W. Śródka, Biomechanical model of the human eyeball, Vol. 91, Oficyna Wydawnicza Politechniki Wrocławskiej, Wrocław 2010.

[13] J. Major, M. Gacek, J.P. Szaflik, Okulistyka 1, 52 (2011).

[14] https://www.glaucomafoundation.org/diagnosing and_treating_glaucoma.htm, 2014.

[15] www.reichert.com/product_details.cfm?skuId=2972\& skuTk=1035455555\#.VW4ĒE9LtlBc, (2014).

[16] http://www.sensimed.ch/en/sensimedtriggerfish/sensimed-triggerfish.html, (2014).

[17] K. Mansouri, R.N. Weinreb, J.H.K. Liu, doi: PLoS ONE 10, 5:e125530 (2015).

[18] http://www.medgadget.com/2013/07/smartcontinuous-monitoring-of-the-intra-ocular-pressurewith-the-triggerfish-contact-lens-qa-with-renegoedkoop-cmo-of-sensimed.html.

[19] H. Lin, S. Godsill, in: Proc. IEEE Workshop on Applications of Signal Processing to Audio and Acoustics, New Paltz 2005, p. 335.

[20] L. Grad, Biuletyn IAiR 31, 79 (2011), (in Polish).

[21] W. Kwiatkowski, Methods for automatic pattern recognition, BEL Studio, Warszawa 2007, (in Polish).

[22] K. Murawski, T. Arciszewski, K. De Jong, Eng. Comput. 16, 275 (2000). 